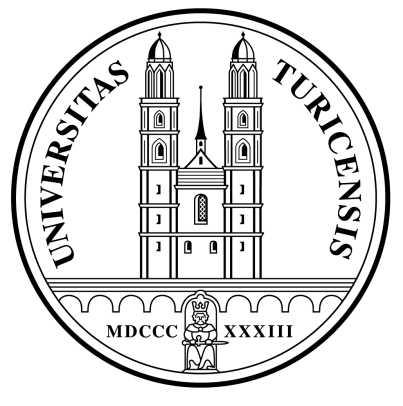

Institute for Empirical Research in Economics

University of Zurich

Working Paper Series

ISSN 1424-0459

Working Paper No. 89

From Rags to Riches:

On Constant Proportions Investment Strategies

Igor V. Evstigneev and Klaus Reiner Schenk-Hoppé

August 2001 


\title{
From Rags to Riches: On Constant Proportions Investment Strategies
}

\author{
Igor V. Evstigneev \\ School of Economic Studies \\ University of Manchester, UK \\ igor.evstigneev@man.ac.uk \\ Klaus Reiner Schenk-Hoppé \\ Institute for Empirical Research in Economics \\ University of Zurich, Switzerland \\ klaus@iew.unizh.ch
}

\begin{abstract}
This paper studies the performance of self-financing constant proportions trading strategies, i.e. dynamic asset allocation strategies that keep a fixed constant proportion of wealth invested in each asset in all periods in time. We prove that any self-financing constant proportions strategy yields a strictly positive exponential rate of growth of investor's wealth in a financial market in which prices are described by stationary stochastic processes and the price ratios are non-degenerate. This result might be regarded as being counterintuitive because any such strategy yields no increase of wealth under constant prices. We further show that the result also holds under small transaction costs, which is important for the viability of this approach, since constant proportions strategies require frequent rebalancing of the portfolio.
\end{abstract}

\section{Introduction}

The problem of optimal portfolio selection is central to any theory of investment in financial markets. While investors' objectives can be manifold, it is often useful to focus on certain optimality criteria as benchmarks. The theory of optimum-growth portfolio, or log-optimum investment, studies portfolio selection rules that maximize the logarithmic growth rate of investor's wealth; see e.g. Algoet and Cover (1988) and the survey by Hakansson and Ziemba (1995). When a strictly positive rate of growth can be achieved, wealth asymptotically becomes unbounded and, in the long run, overtakes any other investment strategy. The log-optimum investment principle - often referred to as the Kelly rule (Kelly 1956) in the case of independent and 
identically distributed returns on investment — has proved quite successful in actual financial markets, Thorp (1971).

One might be tempted to consider self-financing investment strategies that yield exponential growth of wealth as being exceptional and in general difficult to find. However, as we show in this paper, any constant proportions trading strategy yields unbounded and exponentially fast growth of wealth in a stationary financial market, provided the investor trades in at least two stocks. To derive this result we need only a mild assumption of non-degeneracy of the price process. By definition, constant proportions strategies require the investment of a fixed constant proportion of wealth in each asset in all periods in time. These trading strategies are self-financing and only call for a non-zero initial investment; hence investors following this rule go "from rags to riches." This result seems counterintuitive because any constant proportions strategy yields no increase of wealth under constant prices. Stationarity of the financial market rules out any systematic gain from investments, e.g. through increasing prices. However, any persistent stationary variation of prices (not being identical over assets) yields unbounded growth of wealth under every constant proportions strategy.

Constant proportions strategies have been studied-inspired by the optimality properties of the Kelly rule - in many different frameworks, see e.g. Browne and Whitt (1996), Browne (1998), and Aurell, Baviera, Hammarlid, Serva, and Vulpiani (2000).

The issue of transaction costs is quite important for the analysis of optimal investment. In our approach, transaction costs disclose the major drawback of constant proportions strategies - the frequent rebalancing of the portfolio. We take this criticism into account by showing that our result also holds when transactions are costly - provided the costs are sufficiently small.

A substantial role in the paper is played by the notion of a balanced investment strategy. This notion (in a somewhat different form) was first employed in the context of stochastic generalizations of the von Neumann economic growth model by Radner (1971). It was analyzed in a quite general setting by Arnold, Evstigneev, and Gundlach (1999). This approach appears to be new to the financial market literature.

Due to the partial equilibrium character of our analysis, the impression of a money machine might arise and, moreover, it might be conjectured that constant proportions strategies are uninteresting when closing the model and dealing with general equilibrium. This perspective has recently been explored from an evolutionary point of view in a different strand of literature, Blume and Easley (1992), Hens and Schenk-Hoppé (2001), and Evstigneev, Hens, and Schenk-Hoppé (2001). Their findings emphasize the relevance of constant proportions investment strategies.

The next section explains the model without transaction costs. Section 3 presents the main result on unbounded growth of wealth. The model with transaction costs is introduced and analyzed in Section 4. 


\section{Model}

Let an investor observe prices and take actions in discrete periods of time $t=0,1,2, \ldots$. The factors underlying price changes are uncertain, and they are described in probabilistic terms. Uncertainty is modelled by a stochastic process $s_{t}, t=0, \pm 1, \pm 2, \ldots$, taking values in a measurable space $S$. The value of the random parameter $s_{t}$ characterizes the "state of the world" at time $t$.

Consider a financial market with $K \geq 2$ assets whose prices $p_{t}>0$, $t=0,1,2, \ldots$, form a sequence of strictly positive random vectors with values in the $K$-dimensional linear space $R^{K}$. We assume that $p_{t}$ depends on the history of the process $s_{t}$ up to time $t$, i.e.

$$
p_{t}=p_{t}\left(s^{t}\right), s^{t}=\left(\ldots, s_{t-1}, s_{t}\right) .
$$

(All functions of $s^{t}$ considered in what follows are supposed to be measurable.)

At each time period $t$, an investor chooses a portfolio $h_{t}\left(s^{t}\right)=\left(h_{t}^{1}\left(s^{t}\right), \ldots\right.$, $\left.h_{t}^{K}\left(s^{t}\right)\right) \geq 0$, where $h_{t}^{i}$ is the number of units of asset $i$ in the portfolio $h_{t}$. The assumption of non-negativity of $h_{t}$ rules out short sales of the assets in our model. A sequence $h_{t}\left(s^{t}\right), t=0,1,2, \ldots$, specifying a portfolio at each time period $t$ and in every random situation $s^{t}$, is called a trading strategy.

We begin with an analysis of the case of no transaction costs; then we describe changes that are necessary for dealing with situations where transactions are costly.

Given a number $w_{0}>0$, we say that $h_{t}, t=0,1,2, \ldots$, is a trading strategy with initial wealth $w_{0}$ if $p_{0} h_{0}=w_{0}$. A trading strategy is termed self-financing if

$$
p_{t}\left(s^{t}\right) h_{t}\left(s^{t}\right) \leq p_{t}\left(s^{t}\right) h_{t-1}\left(s^{t-1}\right), \quad t=1,2, \ldots \quad \text { (a.s.). }
$$

The inequalities in (1) are supposed to hold almost surely (a.s.) with respect to the probability measure $P$ induced by the stochastic process $s_{t}$, $t=0, \pm 1, \pm 2, \ldots$, on the space of its paths. These inequalities state that the budget constraint, imposing restrictions on the choice of the investor's portfolio in every time period, is determined by the value of the previous period's portfolio at the current prices.

Let us say that the market is stationary if the process $s_{t}$ is stationary and the price vectors $p_{t}$ do not explicitly depend on $t$, i.e., $p_{t}=p\left(s^{t}\right)$. When analyzing such markets, it is of interest to consider trading strategies of balanced growth (or, briefly, balanced strategies). These strategies are of the form

$$
h_{t}\left(s^{t}\right)=\gamma\left(s^{1}\right) \ldots \gamma\left(s^{t}\right) \tilde{h}\left(s^{t}\right), \quad t=1,2, \ldots,
$$

where $\gamma(\cdot)>0$ is a scalar-valued function and $\tilde{h}(\cdot) \geq 0$ is a vector function such that $\ln \gamma\left(s^{t}\right)$ and $\ln \left|\tilde{h}\left(s^{t}\right)\right|$ are integrable with respect to the measure 
$P$ (for a vector $h=\left(h^{i}\right)$, we write $|h|=\sum_{i}\left|h^{i}\right|$ ). In probabilistic terms, integrability of the above functions means finiteness of the expectations

$$
E\left|\ln \gamma\left(s^{t}\right)\right| \text { and } E|\ln | \tilde{h}\left(s^{t}\right)|| .
$$

In the case $t=0$, we assume in (2) that

$$
h_{0}\left(s^{0}\right)=\tilde{h}\left(s^{0}\right) .
$$

The term "balanced" used in the foregoing definition is justified because (2) implies that all ratios

$$
\frac{h_{t}^{i}\left(s^{t}\right)}{h_{t}^{j}\left(s^{t}\right)}=\frac{\tilde{h}^{i}\left(s^{t}\right)}{\tilde{h}^{j}\left(s^{t}\right)}, i \neq j,
$$

describing the proportions between the amounts of different assets in the portfolio, form stationary stochastic processes. Furthermore, the random growth rate of the amount of each asset $i=1, \ldots, K$, in the portfolio

$$
\frac{h_{t}^{i}\left(s^{t}\right)}{h_{t-1}^{i}\left(s^{t-1}\right)}=\gamma\left(s^{t}\right) \frac{\tilde{h}^{i}\left(s^{t}\right)}{\tilde{h}^{i}\left(s^{t-1}\right)}
$$

is a stationary process. Clearly, for a balanced strategy the self-financing condition (1) is equivalent to

$$
\gamma\left(s^{t}\right) p\left(s^{t}\right) \tilde{h}\left(s^{t}\right) \leq p\left(s^{t}\right) \tilde{h}\left(s^{t-1}\right) \quad \text { (a.s.). }
$$

In view of stationarity, if (6) holds for some $t$, then it holds automatically for all $t$. Finally, if every component $p^{k}\left(s^{t}\right), k=1,2, \ldots, K$, of the vector $p\left(s^{t}\right)$ satisfies

$$
E\left|\ln p^{k}\left(s^{t}\right)\right|<\infty,
$$

then we can associate a balanced strategy (2) to any non-negative vector function $\tilde{h}\left(s^{t}\right)$ with $E|\ln | \tilde{h}\left(s^{t}\right)||<\infty$ by defining

$$
\gamma\left(s^{t}\right):=\frac{p\left(s^{t}\right) \tilde{h}\left(s^{t-1}\right)}{p\left(s^{t}\right) \tilde{h}\left(s^{t}\right)} .
$$

For this strategy, relations (1) and (6) hold as equalities.

In the sequel, we will assume that the process $s_{t}, t=0, \pm 1, \pm 2, \ldots$, is ergodic, and the prices $p^{1}\left(s^{t}\right), \ldots, p^{K}\left(s^{t}\right)$ satisfy (7).

Our analysis will be based on the following result. The proposition below shows that, under quite general assumptions, the growth rate of wealth of any investor employing a balanced trading strategy is completely determined by the expected value of $\gamma$. We further show that strict positivity of $E \ln \gamma\left(s^{0}\right) \equiv E \ln \gamma\left(s^{t}\right)$ implies exponential growth of wealth, i.e., $p_{t} h_{t} \rightarrow \infty$ a.s. exponentially fast. 
Proposition 1 For any balanced trading strategy (2), we have

$$
\left.\lim _{t \rightarrow \infty} \frac{1}{t} \ln \left(p_{t} h_{t}\right)=\lim _{t \rightarrow \infty} \frac{1}{t} \ln \left|h_{t}\right|=E \ln \gamma\left(s^{0}\right) \quad \text { (a.s. }\right) .
$$

Proof. We can write

$$
\frac{1}{t} \ln \left|h_{t}\right|=\frac{1}{t} \sum_{m=1}^{t} \ln \gamma\left(s^{m}\right)+\frac{1}{t} \ln \left|\tilde{h}\left(s^{t}\right)\right|,
$$

and so the second equality in (9) is an immediate consequence of the Birkhoff ergodic theorem, since $t^{-1} \ln \left|\tilde{h}\left(s^{t}\right)\right| \rightarrow 0$ by virtue of integrability of $\ln \left|\tilde{h}\left(s^{t}\right)\right|$. The first equality in (9) follows from the relations $\left|\ln \left(p_{t} h_{t}\right)-\ln \right| h_{t}|| \leq$ $\sum_{k}\left|\ln p^{k}\left(s^{t}\right)\right|$ and $(7)$.

The question which appears naturally when dealing with the above model is whether, in the present stationary context, there exist balanced strategies exhibiting possibilities for unbounded growth. Consider, for the moment, the deterministic case, where $S$ consists of a single point. Then the self-financing condition (1) reduces to

$$
p h_{t} \leq p h_{t-1},
$$

with some constant price vector $p>0$ (in the deterministic case, a stationary process is nothing but a constant). A balanced strategy is given by $h_{t}=\gamma^{t} \tilde{h}$, where $\gamma>0$ is a constant scalar and $\tilde{h} \geq 0$ is a constant non-zero vector. We can immediately see from (10) that the maximum possible value for $\gamma$ is 1 , which rules out any possibility of a non-zero growth.

The above deterministic argument totally agrees with our intuition, and it would be natural to expect that it could be extended to the general, stochastic case. However, this intuition fails, and it turns out that, in a stochastic world, one can usually design a variety of balanced strategies exhibiting almost surely unbounded, and even exponential, growth. Moreover, as the results in the next section show, the exponential growth is a typical phenomenon, which can be established for a broad class of balanced strategies described in terms of proportional investment rules. Since the prices of the assets form stationary processes, no dividends are paid, and the strategies we deal with are purely self-financing, this result may look, at the first glance, counterintuitive.

To formalize the idea of proportional investments, we introduce the following definition, which plays a key role in this paper. Let $\lambda=\left(\lambda_{1}, \ldots, \lambda_{K}\right)$ be a vector in the open simplex

$$
\Delta^{K}=\left\{\left(\lambda_{1}, \ldots, \lambda_{K}\right): \lambda_{k}>0, \sum_{k} \lambda_{k}=1\right\} .
$$

We shall say that a trading strategy $h_{t}$ is a constant proportions strategy if

$$
p_{t}^{k} h_{t}^{k}=\lambda_{k} p_{t} h_{t-1}
$$


for all $t=1,2, \ldots$ and $k=1, \ldots, K$. In every period in time, an investor using this strategy rebalances her portfolio by investing the constant share $\lambda_{k}$ of her wealth $p_{t} h_{t-1}$ into the $k$ th asset. The wealth at the beginning of period $t$ is determined by evaluating the portfolio $h_{t-1}$ from the previous period at the current prices $p_{t}^{k}$. Note that a constant proportions strategy is always self-financing because (11) implies $p_{t} h_{t}=p_{t} h_{t-1}$. Also, note that such a strategy is uniquely defined by its initial portfolio $h_{0}\left(s^{0}\right)$ and the vector $\lambda$ : for every $t \geq 1$, the portfolio $h_{t}\left(s^{t}\right)$ can be determined recursively by using equation (11). Therefore we shall say that $h_{t}$ is generated by $\lambda$ and $h_{0}$. In this paper, we deal only with those strategies for which $\lambda_{k}>0$ for all $k$; proportional investment rules of this kind are sometimes termed completely mixed.

Consider two constant proportions strategies $h_{t}$ and $\hat{h}_{t}$ generated by one and the same $\lambda \in \Delta^{K}$ and different initial portfolios $h_{0} \neq 0$ and $\hat{h}_{0} \neq 0$. It follows from (11) that

$$
c h_{t}^{k} \leq \hat{h}_{t}^{k} \leq C h_{t}^{k}, \quad k=1, \ldots, K, t=1,2, \ldots,
$$

where $c$ and $C$ are defined by

$$
c=\frac{\left|\hat{h}_{0}\right|}{\left|h_{0}\right|} \cdot \frac{\min _{n} p_{1}^{n}}{\max _{n} p_{1}^{n}}, \quad C=\frac{\left|\hat{h}_{0}\right|}{\left|h_{0}\right|} \cdot \frac{\max _{n} p_{1}^{n}}{\min _{n} p_{1}^{n}}
$$

with $p_{1}^{n}=p^{n}\left(s^{1}\right)$. Inequalities (12) can be established, by using (11), first for $t=1$ and then for all $t>1$ by way of induction. Relations (12) show that asymptotic properties of constant proportions strategies do not depend on their initial portfolios. This concerns, in particular, the property of exponential growth: we have $\lim t^{-1} \ln \left|h_{t}\right|>0$ a.s. if and only if $\lim t^{-1} \ln \left|\hat{h}_{t}\right|>0$ a.s.

\section{Main Result}

In this section, we state and prove a central result of the paper (Theorem 1). To derive the result, we impose a condition of non-degeneracy of the price process $p\left(s^{t}\right)$ : with positive probability, the variable $p^{k}\left(s^{t}\right) / p^{k}\left(s^{t-1}\right)$ is not constant with respect to $k=1,2, \ldots, K$, i.e., there exist $m$ and $n$ (that might depend on $s^{t}$ ) for which

$$
\frac{p^{m}\left(s^{t}\right)}{p^{m}\left(s^{t-1}\right)} \neq \frac{p^{n}\left(s^{t}\right)}{p^{n}\left(s^{t-1}\right)}
$$

Under this assumption, the following theorem holds.

Theorem 1 Let $\lambda=\left(\lambda_{1}, \ldots, \lambda_{K}\right)$ be a vector in $\Delta^{K}$, and let $w_{0}$ be a strictly positive number. Then there exists a vector function $h_{0}\left(s^{0}\right) \geq 0$ such that the 
constant proportions strategy $h_{t}$ generated by $\lambda$ and $h_{0}$ is a balanced strategy with initial wealth $w_{0}$, and we have

$$
\lim _{t \rightarrow \infty} \frac{1}{t} \ln p_{t} h_{t}=\lim _{t \rightarrow \infty} \frac{1}{t} \ln \left|h_{t}\right|>0 \quad \text { (a.s.). }
$$

Thus, given any vector of proportions $\lambda \in \Delta^{K}$ and an initial wealth $w_{0}>$ 0 , we can construct an initial portfolio $h_{0}$ satisfying the budget constraint $p_{0} h_{0}=w_{0}$ so that the constant proportions strategy $h_{t}$ defined recursively by (11) turns out to be balanced and exhibits exponential growth. Recall that this strategy is automatically self-financing by virtue of (11). As has been noticed at the end of the previous section, the property of exponential growth of $h_{t}$ does not depend on the initial portfolio, and will retain if we replace $h_{0}$ by any other $\hat{h}_{0} \neq 0$. (However, the strategy $h_{t}$ might become non-balanced under such a replacement).

Proof of Theorem 1. Define

$$
\tilde{h}\left(s^{t}\right)=\left(\frac{\lambda_{1} w_{0}}{p^{1}\left(s^{t}\right)}, \ldots, \frac{\lambda_{K} w_{0}}{p^{K}\left(s^{t}\right)}\right)
$$

and

$$
\gamma\left(s^{t}\right)=\frac{p\left(s^{t}\right) \tilde{h}\left(s^{t-1}\right)}{w_{0}}\left[=\sum_{k=1}^{K} \lambda_{k} \frac{p^{k}\left(s^{t}\right)}{p^{k}\left(s^{t-1}\right)}\right] .
$$

By virtue of (7), the expectations (3) are finite. Consider the balanced strategy $h_{t}$ defined by (2) and (4), where $\tilde{h}$ and $\gamma$ are specified in (15), (16). For each $t=0,1, \ldots$, we have

$$
\begin{aligned}
p^{k}\left(s^{t+1}\right) h_{t+1}^{k}\left(s^{t+1}\right) & =p^{k}\left(s^{t+1}\right) \gamma\left(s^{1}\right) \ldots \gamma\left(s^{t+1}\right) \frac{\lambda_{k} w_{0}}{p^{k}\left(s^{t+1}\right)} \\
& =\gamma\left(s^{1}\right) \ldots \gamma\left(s^{t}\right) \cdot \gamma\left(s^{t+1}\right) \lambda_{k} w_{0} \\
& =\gamma\left(s^{1}\right) \ldots \gamma\left(s^{t}\right) \cdot p\left(s^{t+1}\right) \tilde{h}\left(s^{t}\right) \lambda_{k}=\lambda_{k} p\left(s^{t+1}\right) h_{t}\left(s^{t}\right) .
\end{aligned}
$$

Thus $h_{t}$ coincides with the constant proportions strategy generated by $\lambda=$ $\left(\lambda_{1}, \ldots, \lambda_{K}\right)$ and $h_{0}=\tilde{h}\left(s^{0}\right)$, and, furthermore, $p\left(s^{0}\right) h_{0}\left(s^{0}\right)=p\left(s^{0}\right) \tilde{h}\left(s^{0}\right)=$ $\sum_{k} \lambda_{k} w_{0}=w_{0}$.

In view of Proposition 1, it remains to show that $E \ln \gamma\left(s^{t}\right)>0$. By virtue of Jensen's inequality (which is applicable since $\lambda_{k}>0$ and $\sum \lambda_{k}=1$ ), we have

$$
\ln \sum_{k=1}^{K} \lambda_{k} \frac{p^{k}\left(s^{t}\right)}{p^{k}\left(s^{t-1}\right)} \geq \sum_{k=1}^{K} \lambda_{k} \ln \frac{p^{k}\left(s^{t}\right)}{p^{k}\left(s^{t-1}\right)},
$$

and, in view of assumption (13), the probability that this inequality is strict is greater than zero. The number $E \ln \gamma\left(s^{t}\right)$ is equal to the expected value of the expression on the left-hand side of (17). Consequently,

$$
E \ln \gamma\left(s^{t}\right)>\sum_{k=1}^{K} \lambda_{k} E \ln \frac{p^{k}\left(s^{t}\right)}{p^{k}\left(s^{t-1}\right)}=0
$$


by virtue of stationarity of $s_{t}$ and finiteness of $E\left|\ln p^{k}\left(s^{t}\right)\right|$.

Let us explain the intuition behind this result. Any constant proportions strategy 'exploits' the persistent fluctuation of prices in the following way. Keeping a fixed fraction of wealth invested in each asset implies that after a change in prices an investor sells those assets that are expensive relative to the other assets and purchases relatively cheap assets. The stationarity of prices implies that this portfolio rule yields a strictly positive expected rate of growth, despite the fact that each asset price has growth rate zero. Thereby investors go "from rags to riches."

\section{Transaction Costs}

This section extends the result of the previous section to markets with transaction costs. Transaction costs represent the main obstacle in getting "from rags to riches" when using constant proportions strategies. This is due to the fact that their main disadvantage - the need to frequently rebalance the portfolio-becomes apparent when rebalancing is costly. However, since investors' wealth grows exponentially fast in the absence of transaction costsas shown in Theorem 1, there should be room for small losses in every period (resulting from transactions) without eliminating the possibility of unbounded growth of wealth.

When transaction costs are present in the financial market, the selffinancing condition (1) becomes

$$
p_{t} h_{t}+\sum_{k=1}^{K} \delta_{k} p_{t}^{k}\left|h_{t}^{k}-h_{t-1}^{k}\right| \leq p_{t} h_{t-1} \quad \text { (a.s.) }
$$

for all $t=1,2, \ldots$. (We write $p_{t}^{k}=p^{k}\left(s^{t}\right)$ and $h_{t}^{k}=h_{t}^{k}\left(s^{t}\right)$.) According to (18), the cost of transactions involving asset $k$ is a fixed fraction $\delta_{k} \geq 0$ of the order volume. For simplicity of presentation, we assume that these fractions are the same for buying and selling.

We generalize the previous definition (11) by calling a trading strategy $h_{t}$ a constant proportions strategy associated with a vector $\lambda=\left(\lambda_{1}, \ldots, \lambda_{K}\right) \in \Delta^{K}$ if

$$
p_{t}^{k} h_{t}^{k}=\lambda_{k}\left[p_{t} h_{t-1}-\sum_{n=1}^{K} \delta_{n} p_{t}^{n}\left|h_{t}^{n}-h_{t-1}^{n}\right|\right] \quad \text { (a.s.) }
$$

for all $t=1,2, \ldots$ and $k=1,2, \ldots, K$. By virtue of this definition, the investment (evaluated in terms of the market price $p_{t}$ ) in asset $k$ in every time period is equal to the fraction $\lambda_{k}$ of the beginning-of-period wealth $p_{t} h_{t-1}$ less the total transaction costs. Clearly (19) implies (18), so that any strategy satisfying (19) is self-financing.

If there are no transaction costs, i.e. $\delta_{n}=0$ for all $n=1, \ldots, K$, then (19) coincides with equation (11). The latter equation allows to construct a 
constant proportions strategy recursively, based on the knowledge of $\lambda$ and $h_{0}$. A similar construction can be performed when transaction costs are present as the proposition below shows.

Proposition 2 Let $\lambda \in \Delta^{K}$, and let $\delta_{1}, \ldots, \delta_{K}$ be nonnegative numbers such that $\delta_{k}<1$ for all $k$. Then, for every $h_{t-1} \geq 0$, there is a unique vector $h_{t}=\left(h_{t}^{1}, \ldots, h_{t}^{K}\right)$ satisfying (19). For this vector, we have

$$
h_{t}^{k}=\frac{\lambda_{k}}{p_{t}^{k}} \beta,
$$

where $\beta$ is a unique non-negative solution to the equation

$$
\beta+\sum_{n=1}^{K} \delta_{n}\left|\lambda_{n} \beta-p_{t}^{n} h_{t-1}^{n}\right|=p_{t} h_{t-1} .
$$

Consequently, in the model with transaction costs $\delta_{k}<1$ as well as in the model without transaction costs, we can speak of constant proportions strategies generated by a vector of proportions $\lambda$ and an initial portfolio $h_{0}$.

Before proving the above proposition, we formulate the main result of this section.

Theorem 2 Let $\lambda=\left(\lambda_{1}, \ldots, \lambda_{K}\right) \in \Delta^{K}$, and let $w_{0}>0$. Then there exists a number $\varepsilon>0$ for which the following assertion holds. In the model with transaction costs $\delta_{1}, \ldots, \delta_{K} \geq 0$ not exceeding $\varepsilon$, one can construct a portfolio $h_{0}\left(s^{0}\right) \geq 0$ such that the constant proportions strategy $h_{t}$ generated by $\lambda$ and $h_{0}$ is a balanced strategy with initial wealth $w_{0}$, growing with an exponential rate: $\lim _{t \rightarrow \infty} t^{-1} \ln p_{t} h_{t}=\lim _{t \rightarrow \infty} t^{-1} \ln \left|h_{t}\right|>0$ (a.s.).

Thus conclusions analogous to those obtained in the previous section can be established for a model with transaction costs, provided these costs are small enough. We will prove Theorem 2, by using a "small perturbation" technique, showing that the original model may be viewed as a limit of the perturbed one as $\delta=\left(\delta_{1}, \ldots, \delta_{K}\right) \rightarrow 0$. We first prove Proposition 2 and then turn to Theorem 2.

Proof of Proposition 2. We can see that $h_{t}=\left(h_{t}^{k}\right)_{k=1, \ldots, K} \geq 0$ is a solution to (19) if and only if, for some $\beta \geq 0$, relations (20) and (21) hold. Thus it suffices to show that (21) has a unique solution $\beta \geq 0$. Denote the expressions on the left-hand side and on the right-hand side of (21) by $f(\beta)$ and $\alpha$, respectively. The function $f(\beta)$ is continuous on $[0, \infty)$, and we have $f(0) \leq \alpha$ (since $\delta_{k}<1$ ) whereas $f(\beta)>\alpha$ for all $\beta$ large enough. Therefore at least one solution of the equation $f(\beta)=\alpha$ exists. To verify that this solution is unique observe that $f(\beta)$ is strictly increasing on $(0, \infty)$. Indeed, $f$ is continuously differentiable in $\beta$ at all points in $(0, \infty)$ except for $\beta=p_{t}^{n} h_{t-1}^{n} / \lambda_{n}, n=1, \ldots, K$, and at all points at which the derivative exists, it is bounded below by $1-\sum_{n} \lambda_{n} \delta_{n}>0$. 
Remark 1 In the proof of Theorem 2 we will need the following inequalities for the solution $\beta$ to the equation (21):

$$
\min _{n}\left(p_{t}^{n} h_{t-1}^{n}\right) \leq \beta \leq p_{t} h_{t-1} .
$$

The latter inequality is an immediate consequence of (21). Suppose the former is not true, i.e., $p_{t}^{n} h_{t-1}^{n}>\beta$ for all $n$. Then (21) yields

$$
\beta-\sum_{n=1}^{K} \delta_{n}\left(\lambda_{n} \beta-p_{t}^{n} h_{t-1}^{n}\right)=p_{t} h_{t-1},
$$

and so

$$
\beta=\frac{\sum\left(1-\delta_{n}\right) p_{t}^{n} h_{t-1}^{n}}{1-\sum \delta_{n} \lambda_{n}}=\frac{\sum\left(1-\delta_{n}\right) p_{t}^{n} h_{t-1}^{n}}{\sum\left(1-\delta_{n}\right) \lambda_{n}} \geq \min _{n} \frac{p_{t}^{n} h_{t-1}^{n}}{\lambda_{n}} \geq \min _{n}\left(p_{t}^{n} h_{t-1}^{n}\right),
$$

which proves the first inequality in (22).

Proof of Theorem 2. Assume $\delta_{k}<1, k=1,2, \ldots, K$. As in the proof of Theorem 1, we set $\tilde{h}\left(s^{t}\right)=\left(w_{0} \lambda_{k} / p^{k}\left(s^{t}\right)\right)_{k=1, \ldots, K}$. Further, we define $\gamma=\gamma\left(s^{t}\right)$ as the non-negative solution to the equation

$$
\gamma+\sum_{n=1}^{K} \delta_{n}\left|\lambda_{n} \gamma-p_{t}^{n} \frac{\tilde{h}_{t-1}^{n}}{w_{0}}\right|=p_{t} \frac{\tilde{h}_{t-1}}{w_{0}},
$$

where $p_{t}^{n}=p^{n}\left(s^{t}\right)$ and $\tilde{h}_{t}=\tilde{h}\left(s^{t}\right)$. By virtue of Proposition 2, this solution exists and is unique. (If $\delta_{n}=0$ for all $n=1, \ldots, K$, then $\gamma\left(s^{t}\right)$ coincides with the function in (16).) From (7), we can see that $E|\ln | \tilde{h}\left(s^{t}\right)||<\infty$. By virtue of inequalities (22), we have

$$
\min _{n}\left[\lambda_{n} \frac{p^{n}\left(s^{t}\right)}{p^{n}\left(s^{t-1}\right)}\right] \leq \gamma\left(s^{t}\right) \leq \sum_{n} \lambda_{n} \frac{p\left(s^{t}\right)}{p\left(s^{t-1}\right)},
$$

and so $E\left|\ln \gamma\left(s^{t}\right)\right|<\infty$. Thus the functions $\tilde{h}(\cdot)$ and $\gamma(\cdot)$ just constructed define a balanced strategy $h_{t}=\gamma\left(s^{1}\right) \ldots \gamma\left(s^{t}\right) \tilde{h}\left(s^{t}\right), h_{0}=\tilde{h}\left(s^{0}\right)$.

Let us prove that $h_{t}$ coincides with the constant proportions strategy generated by $h_{0}=\tilde{h}\left(s^{0}\right)$ and $\lambda$. To this end we have to verify (19), or, equivalently, to show that (20) and (21) hold for some $\beta$. Define $\beta=\gamma\left(s^{1}\right) \ldots \gamma\left(s^{t}\right) w_{0}$. Then we have

$$
h_{t}^{k}=\gamma\left(s^{1}\right) \ldots \gamma\left(s^{t}\right) \tilde{h}^{k}\left(s^{t}\right)=\gamma\left(s^{1}\right) \ldots \gamma\left(s^{t}\right) \frac{w_{0} \lambda_{k}}{p^{k}\left(s^{t}\right)}=\frac{\lambda_{k}}{p_{t}^{k}} \beta,
$$

which proves (20). Finally, (21) is equivalent to the equation

$$
\gamma\left(s^{t}\right) w_{0}+\sum_{n=1}^{K} \delta_{n}\left|\lambda_{n} \gamma\left(s^{t}\right) w_{0}-p_{t}^{n} \tilde{h}_{t-1}^{n}\right|=p_{t} \tilde{h}_{t-1},
$$


holding by virtue of (23).

Note that the function $\gamma$ involved in the construction of the balanced strategy $h_{t}$ depends on the vector of transaction $\operatorname{costs} \delta$. For the sake of clarity, let us denote it by $\gamma_{\delta}$. By the definition of $\gamma_{\delta}\left(s^{t}\right)$ (see (23)), we have $\gamma_{\delta}\left(s^{t}\right) \rightarrow \gamma_{0}\left(s^{t}\right)$ as $\delta \rightarrow 0$. It follows from (24) and (7) that there exists an integrable function $\theta\left(s^{t}\right)$ such that $\left|\ln \gamma_{\delta}\left(s^{t}\right)\right| \leq \theta\left(s^{t}\right)$. Therefore we can apply Lebesgue's dominated convergence theorem, which yields $\lim _{\delta \rightarrow 0} E \ln \gamma_{\delta}\left(s^{t}\right)=$ $E \ln \gamma_{0}\left(s^{t}\right)$. From Theorem 1 we know that, for $\delta=0, E \ln \gamma_{0}\left(s^{t}\right)>0$. Consequently, $E \ln \gamma_{\delta}\left(s^{t}\right)>0$ for all $\delta$ such that $|\delta|<\varepsilon$, where $\varepsilon>0$ is a sufficiently small number. To complete the proof it suffices to employ Proposition 1.

\section{References}

Algoet, P. H., And T. M. Cover (1988): "Asymptotic Optimality and Asymptotic Equipartition Properties of Log-Optimum Investment," Annals of Probability, 16, 876-898.

Arnold, L., I. V. Evstigneev, and V. M. Gundlach (1999): "ConvexValued Random Dynamical Systems: A Variational Principle for Equilibrium States," Random Operators and Stochastic Equations, 7, 23-28.

Aurell, E., R. Baviera, O. Hammarlid, M. Serva, and A. Vulpiani (2000): "A general methodology to price and hedge derivatives in incomplete markets," International Journal of Theoretical and Applied Finance, $3,1-24$.

Blume, L., And D. Easley (1992): "Evolution and Market Behavior," Journal of Economic Theory, 58, 9-40.

Browne, S. (1998): "The Return on Investment from Proportional Portfolio Strategies," Advances in Applied Probability, 30, 216-238.

Browne, S., and W. Whitt (1996): "Portfolio Choice and the Bayesian Kelly Criterion," Advances in Applied Probability, 28, 1145-1176.

Evstigneev, I. V., T. Hens, and K. R. Schenk-Hoppé (2001): "Market selection of financial trading strategies: Global stability," Working Paper No. 83, Institute for Empirical Research in Economics, University of Zurich.

Hakansson, N. H., And W. T. Ziemba (1995): "Capital Growth Theory," in Handbooks in Operations Research and Management Science, Volume 9, Finance, ed. by R. A. Jarrow, V. Maksimovic, and W. T. Ziemba, chap. 3, pp. 65-86. Elsevier, Amsterdam. 
Hens, T., And K. R. Schenk-Hoppé (2001): "An Evolutionary Portfolio Theory," Working Paper No. 74, Institute for Empirical Research in Economics, University of Zurich.

Kelly, J. (1956): "A New Interpretation of Information Rate," Bell System Technical Journal, 35, 917-926.

RADNeR, R. (1971): "Balanced Stochastic Growth at the Maximum Rate," in Contributions to the von Neumann Growth Model (Zeitschrift für Nationalökonomie, Suppl. 1), ed. by G. Bruckman, and W. Weber, pp. 39-62. Springer-Verlag, Vienna.

Thorp, E. (1971): "Portfolio Choice and the Kelly Criterion," in Stochastic Models in Finance, ed. by W. Ziemba, and R. Vickson, pp. 599-619. Academic Press, New York. 